\title{
Multiple Regression \\ - Artikel Nr. 13 der Statistik-Serie in der DMW -
}

\section{Multiple regression}

Autoren

Institut

\section{R. Bender ${ }^{1}$ A. Ziegler $^{2}$ S. Lange}

Institut für Qualität und Wirtschaftlichkeit im Gesundheitswesen, Köln

2 Institut für Medizinische Biometrie und Statistik, Universitätsklinikum Schleswig-Holstein, Campus Lübeck, Universität zu Lübeck
Schlüsselwörter

Lineare Regression

Verzerrung

Regressionskoeffizient

Modellgüte

Key words

Linear regression

$\checkmark$ Bias

Regression coefficient

Goodness-of-fit
Bibliografie

DOI 10.1055/s-2007-959036 Dtsch Med Wochenschr 2007; 132: e30-e32 - (c) Georg Thieme Verlag KG Stuttgart - New York . ISSN 0012-0472

Korrespondenz

Privatdozent Dr. rer. biol. hum. Ralf Bender

Institut für Qualität und Wirtschaftlichkeit im Gesundheitswesen (IQWiG)

Dillenburger Straße 27

51105 Köln

eMail Ralf.Bender@iqwig.de

\section{Einfache lineare Regression \\ $\nabla$}

Mit Hilfe der einfachen linearen Regression (engl.: simple linear regression) lässt sich der Zusammenhang zwischen zwei stetigen Merkmalen statistisch untersuchen [6]. Hierbei wird unterschieden zwischen der erklärenden Variable X ( $\mathrm{z}$. B. $X=$ Gewicht in $\mathrm{kg}$ ) und der Zielvariable $Y(z$. B. $\mathrm{Y}=$ systolischer Blutdruck in $\mathrm{mm} \mathrm{Hg}$ ). Der Zusammenhang wird mit Hilfe der Geradengleichung

$Y=\alpha+\beta X$

untersucht, d. h. man beschränkt sich auf die Untersuchung linearer Zusammenhänge. Ist die Annahme der Linearität verletzt, d. h. liegen die Punkte $(X, Y)$ im Mittel gar nicht auf einer Geraden, müssen die Variablen entweder so transformiert werden, dass zwischen den transformierten Variablen ein linearer Zusammenhang besteht, oder es muss ein entsprechendes nichtlineares Regressionsmodell angewendet werden. Der Parameter von Interesse ist i. d. R. der Regressionskoeffizient $\beta$; er gibt den Anstieg von $Y$ bei einem Anstieg von $X$ um eine Einheit an: je größer der Betrag von $\beta$ ist, desto größer ist der Einfluss von $\mathrm{X}$ auf $\mathrm{Y}$. Der Achsenabschnitt (engl.: intercept) $\alpha$ gibt den Y-Wert bei $\mathrm{X}=0 \mathrm{an}$. Im obigen Beispiel bedeutet $\beta=1,31$, dass im Mittel mit jedem Anstieg des Gewichts um 1 kg der systolische Blutdruck um 1,31 mm $\mathrm{Hg}$ ansteigt [6].

\section{Multiple lineare Regression \\ $\nabla$}

Das einfache lineare Regressionsmodell lässt sich formal leicht auf ein multiples Modell mit mehreren erklärenden Variablen $\mathrm{X}_{1}, \ldots, \mathrm{X}_{\mathrm{m}}$ verallgemeinern durch

$\mathrm{Y}=\alpha+\beta_{1} \mathrm{X}_{1}+\ldots+\beta_{\mathrm{m}} \mathrm{X}_{\mathrm{m}}$
Mit Hilfe dieses Modells der multiplen linearen Regression (engl.: multiple linear regression) lässt sich der gemeinsame lineare Einfluss der erklärenden Variablen $\mathrm{X}_{1}, \ldots, \mathrm{X}_{\mathrm{m}}$ auf die Zielvariable $\mathrm{Y}$ untersuchen. Dieses allgemeine Modell hat in der medizinischen Statistik eine große Bedeutung, da sehr viele Fragestellungen zur Anwendung multipler Regressionsmodelle führen.

Zunächst einmal sind reine bivariate Zusammenhänge in der medizinischen Forschung eher die Ausnahme. Zwar besteht ein Zusammenhang zwischen dem Gewicht als erklärender Variable $\mathrm{X}$ und dem systolischen Blutdruck als Zielvariable Y, aber in aller Regel gibt es weitere Variablen, die einen Einfluss auf $Y$ haben, z. B. $X_{2}=$ Alter, $\mathrm{X}_{3}=$ Geschlecht und $\mathrm{X}_{4}=$ Rauchen. Durch ein multiples lineares Regressionsmodell lässt sich also der gemeinsame Einfluss der Variablen Gewicht, Alter, Geschlecht und Rauchen auf dem systolischen Blutdruck untersuchen. Zu beachten ist hierbei, dass in die Modellgleichung nur erklärende Variablen mit stetigem und/oder binärem Messniveau betrachtet werden können. Erklärende kategorielle Variablen mit mehr als zwei Kategorien können durch Kodierungstechniken berücksichtigt werden. Am häufigsten werden die Variablen mit Hilfe der so genannten Dummy-Kodierung berücksichtigt. Das bedeutet, dass man eine Kategorie als Referenzkategorie wählt und die anderen Kategorien im Vergleich hierzu als binäre Variablen darstellt. Auf diese Weise lässt sich der Einfluss einer erklärenden Variable mit k Kategorien durch k-1 Regressionskoeffizienten darstellen.

In vielen Anwendungen der medizinischen Statistik interessiert man sich zwar zunächst nur für den Einfluss einer erklärenden Variable $\mathrm{X}_{1}$ auf die Zielvariable $Y$, jedoch muss man andere Variablen im Modell berücksichtigen, um den Zusammenhang zwischen $\mathrm{X}_{1}$ und $\mathrm{Y}$ möglichst unverzerrt schätzen zu können. Ein häufiges Beispiel ist der 
Vergleich von 2 Gruppen (z. B. exponierte und nicht exponierte Personen) bezüglich der Zielvariable $\mathrm{Y}$ (wie bisher $\mathrm{Z}$. B. $\mathrm{Y}=$ systolischer Blutdruck) in einer Beobachtungsstudie. Wenn die Gruppenzugehörigkeit nicht durch Randomisierung zugewiesen werden konnte, kann man nicht davon ausgehen, dass alle weiteren für Y wichtigen erklärenden Variablen in den Gruppen gleich verteilt sind. Würde man einfach den $\boldsymbol{t}$-Test [7] zum Vergleich der Gruppen anwenden, könnte ein signifikanter Unterschied zwischen den Gruppen sowohl auf einen Effekt der Exposition, als auch auf systematische Unterschiede zwischen den beiden Gruppen bezüglich anderer Variablen (z. B. Alter, Geschlecht und Rauchen) zurückzuführen sein. Um eine solche Verzerrung (engl.: bias) bei der Schätzung des Expositionseffekts zu reduzieren (im Idealfall auf Null), müssen die wichtigen, d. h. die prognostisch relevanten Einflussvariablen berücksichtigt werden. Dies ist, als Erweiterung des t-Tests, mit Hilfe eines multiplen Regressionsmodells möglich, in dem die erklärenden Variablen $\mathrm{X}_{1}=$ Exposition, $\mathrm{X}_{2}=$ Alter, $\mathrm{X}_{3}=$ Geschlecht und $\mathrm{X}_{4}=$ Rauchen gemeinsam in einem Modell betrachtet werden. Durch ein solches Modell erhält man den interessierenden Expositionseffekt durch den Regressionskoeffizienten $\beta_{1}$. Da im multiplen Modell die anderen erklärenden Variablen und damit mögliche systematische Unterschiede bezüglich dieser Variablen berücksichtigt sind, spricht man hier von einem nach Alter, Geschlecht und Rauchen adjustierten Regressionskoeffizienten.

Eine solche multifaktorielle Analyse kann in Interventionsstudien kein Ersatz für eine Randomisierung sein. Die Berechnung adjustierter Effekte stellt aber in Fällen, in denen aus ethischen oder praktischen Gründen keine Randomisierung durchgeführt werden kann, eine wesentlich adäquatere Auswertungsstrategie dar als die einfache Schätzung der rohen nicht adjustierten Effekte.

\section{Beispiel: Effektivität eines ambulanten Gewichts- reduktionsprogramms}

In einer Beobachtungsstudie zur Effektivität eines ambulanten Gewichtsreduktionsprogramms wurde anhand einer Stichprobe von $n=294$ übergewichtigen Patienten untersucht, welche Faktoren mit einer Gewichtsabnahme assoziiert sind [5]. Eine Fragestellung war, ob die Gewichtsabnahme bei Männern und Frauen unterschiedlich ist. Als Zielvariable wurde die relative Gewichtsänderung zwischen Therapieende und Therapieanfang in \%

\section{$Y=100 \times($ Gewicht am Ende - Anfangsgewicht $) /$ Anfangsgewicht}

betrachtet, $d$. h. bei negativen Werten für Y liegt eine Gewichtsabnahme vor. Es zeigte sich, dass Männer im Durchschnitt (-8,83\%) mehr abgenommen haben als Frauen (-7,16\%). Der Unterschied von $-1,67 \%$ ist aber nicht signifikant $(\boldsymbol{t}$-Test: $p=0,0731$ ). Genau das gleiche Resultat erhält man, indem eine einfache lineare Regression mit der binären erklärenden Variable Geschlecht ( $1=$ männlich, $0=$ weiblich $)$ durchgeführt wird. Der Regressionskoeffizient entspricht dann gerade der mittleren Differenz zwischen Männern und Frauen (Tab. 1).

Die Formulierung als Regressionsmodell hat den Vorteil, dass es sich auf den Fall mehrerer erklärender Variablen verallgemeinern lässt. Potenzielle erklärende Variablen für die Gewichtsab-
Tab. 1 Einfache lineare Regressionsanalyse für die Assoziation zwischen prozentualer Gewichtsabnahme und Geschlecht bei 294 übergewichtigen Patienten.

\begin{tabular}{|lllll} 
& $\begin{array}{l}\text { Regressions- } \\
\text { koeffizient }\end{array}$ & $\begin{array}{l}\text { Standard- } \\
\text { fehler }\end{array}$ & $\begin{array}{l}\text { 95\% Konfidenz- } \\
\text { intervall }\end{array}$ & p-Wert \\
\hline Achsenabschnitt & $-7,158$ & 0,473 & & 0,0001 \\
\hline $\begin{array}{l}\text { Geschlecht } \\
\text { (männl. vs. weibl.) }\end{array}$ & $-1,675$ & 0,931 & $-3,50$ bis $+0,15$ & 0,0731 \\
\hline
\end{tabular}

Tab. 2 Multiple lineare Regressionsanalyse für die Assoziationen zwischen prozentualen Gewichtsabnahme und Geschlecht, Behandlungsdauer und Bildungsstand bei 294 übergewichtigen Patienten.

\begin{tabular}{|c|c|c|c|c|}
\hline & $\begin{array}{l}\text { Regressions- } \\
\text { koeffizient }\end{array}$ & $\begin{array}{l}\text { Standard- } \\
\text { fehler }\end{array}$ & $\begin{array}{l}\text { 95\% Konfidenz- } \\
\text { intervall }\end{array}$ & $p$-Wert \\
\hline Achsenabschnitt & $-3,152$ & 0,594 & & 0,0001 \\
\hline $\begin{array}{l}\text { Geschlecht } \\
\text { (männl. vs. weibl.) }\end{array}$ & $-2,416$ & 0,819 & $-4,02$ bis $-0,81$ & 0,0034 \\
\hline $\begin{array}{l}\text { Behandlungsdauer } \\
\text { (Monate) }\end{array}$ & $-0,530$ & 0,059 & $-0,65$ bis $-0,41$ & 0,0001 \\
\hline $\begin{array}{l}\text { Bildungsstand } \\
\text { (hoch vs. niedrig) }\end{array}$ & $-4,566$ & 1,886 & $-8,26$ bis $-0,87$ & 0,0161 \\
\hline
\end{tabular}

nahme sind hier u. a. die Dauer der Behandlung und der Bildungsstand. Der Einfachheit halber beschränken wir uns in diesem Beispiel auf die Betrachtung dieser Variablen. Die Berücksichtigung der Behandlungsdauer ist hier besonders wichtig, da diese Variable einen starken Einfluss auf die Gewichtsabnahme besitzt und bei Männern und Frauen unterschiedlich verteilt ist. Während Männer im Mittel 5,8 Monate am Programm teilnahmen, lag diese Zahl bei Frauen im Mittel bei 7,2 Monaten. Da die Behandlungsdauer mit einer höheren Gewichtsabnahme assoziiert ist, ergibt sich bei der einfachen Betrachtung des Unterschieds zwischen Männern und Frauen ein Bias. Dieser kann durch ein adäquates multiples Modell ausgeglichen werden. In einer multiplen linearen Regression mit den erklärenden Variablen Geschlecht, Behandlungsdauer (in Monaten) und Bildungsstand $(1=$ hoch, $0=$ niedrig $)$ zeigt sich ein signifikanter Einfluss des Geschlechts (Tab. 2).

Der nach Behandlungsdauer und Bildungsstand adjustierte durchschnittliche Unterschied zwischen Männern und Frauen ist identisch mit dem Regressionskoeffizienten des Geschlechts $(-2,42 \%, p=0,0034)$, der deutlich höher ist als der rohe nicht adjustierte Unterschied. Durch ein multiples Regressionsmodell lassen sich auch adjustierte Mittelwerte für die einzelnen Gruppen schätzen. Bei gleicher Behandlungsdauer und gleichem Bildungsstand beträgt die relative Gewichtsveränderung bei Männern im Mittel -9,383\% und bei Frauen -6,967\%; die Differenz dieser beiden Werte ergibt gerade den Wert des Regressionskoeffizienten.

\section{Modellbilldung und Modellgüte} $\nabla$

Die sinnvolle Anwendung der multiplen Regressionsanalyse in der Praxis ist sehr viel komplizierter als hier in Kürze dargestellt werden kann. Außer der Auswahl der Zielvariablen und der erklärenden Variablen sollte zunächst eine konkrete Modellgleichung entwickelt werden, welche die untersuchten Zusammen- 
hänge adäquat beschreibt. Dazu gehört die Betrachtung von möglichen Transformationen sowohl der Zielvariablen als auch der erklärenden Variablen, die Untersuchung möglicher nichtlinearer Zusammenhänge durch quadratische oder kubische Effekte und Überlegungen zu möglichen Wechselwirkungen (engl.: interactions) zwischen den erklärenden Variablen. Zur Modellbildung und Untersuchung der Modellgüte (engl.: goodness-of-fit) gibt es eine Reihe von Verfahren, die als Regressionsdiagnostiken (engl.: regression diagnostics) bezeichnet werden. Auf diese Methoden kann im Rahmen dieses Artikel nicht eingegangen werden. Der interessierte Leser sei auf die Literatur verwiesen $[3,4,8]$.

Ein Maß für den prädiktiven Wert eines multiplen linearen Regressionsmodells ist das multiple Bestimmtheitsmaß $\mathbf{R}^{2}$ (engl.: coefficient of determination). Es stellt für die Untersuchung von Zusammenhängen zwischen mehr als zwei Variablen eine Verallgemeinerung des quadrierten Korrelationskoeffizienten [6] dar. Das Bestimmtheitsmaß $\mathrm{R}^{2}$ gibt den Anteil der Varianz der Zielvariablen an, der durch alle erklärenden Variablen im multiplen Regressionsmodell gemeinsam erklärt werden kann. Im betrachteten Beispiel der Assoziationen zwischen relativer Gewichtsabnahme und den erklärenden Variablen Geschlecht, Behandlungsdauer und Bildungsstand ergibt sich der Wert $\mathrm{R}^{2}=0,25$, d. h. durch alle 3 Faktoren gemeinsam lässt sich $25 \%$ der Variabilität der Gewichtsabnahme erklären. Ein großer Anteil der Variabilität wird durch andere Faktoren erklärt, so dass sich die Gewichtsabnahme eines übergewichtigen Patienten aus der Kenntnis der 3 erklärenden Variablen vermutlich nicht mit genügender Genauigkeit ableiten lässt.

Ein limitierender Faktor bei der Anwendung multipler Regressionsmodelle in der Praxis ist häufig der Stichprobenumfang. Einerseits müssen in der Regressionsgleichung alle wichtigen erklärenden Variablen enthalten sein, andererseits benötigt man mit steigender Zahl der erklärenden Variablen auch größere Stichproben. Der benötigte Stichprobenumfang hängt natürlich immer von der konkreten Situation ab. Als Faustregel gilt jedoch, dass man in einer multiplen linearen Regression pro Modellparameter mindestens 10 Beobachtungen benötigt, um ein einigermaßen stabiles Modell zu erhalten [4].

\section{Übersicht über Regressionsmethoden \\ $\nabla$}

Die multiple lineare Regression ist eine spezielle Klasse der Regressionsmethoden, die in Frage kommt, wenn die betrachtete Zielvariable stetiges Messniveau besitzt. Je nach Zahl und Messniveau der erklärenden Variablen lassen sich auch der $\boldsymbol{t}$-Test [7] und die Methoden der Varianzanalyse [1] in die Klasse der linearen Regression einbetten. Ein lineares Regressionsmodell mit genau einer erklärenden Variablen mit binärem Messniveau ist äquivalent zum $\boldsymbol{t}$-Test (Vergleich von 2 Gruppen). Liegt eine erklärende Variable mit nominalem Messniveau (Vergleich mehrerer Gruppen) vor, ergibt sich das Varianzanalysemodell der Einfachklassifikation. Bei mehreren nominal skalierten erklärenden Variablen, erhält man die Varianzanalysemodelle der Mehrfachklassifikation.

Hat die betrachtete Zielvariable kein stetiges Messniveau, so kann die Klasse der linearen Regressionsmodelle nicht sinnvoll angewendet werden. Bei binären Zielvariablen (Ereignis ja/

\section{Tab. 3 Übersetzung (deutsch - englisch)}

\begin{tabular}{|ll}
\hline einfache lineare Regression & simple linear regression \\
\hline erklärende Variable & explanatory variable \\
\hline Zielvariable & response variable \\
\hline Regressionskoeffizient & regression coefficient \\
\hline Achsenabschnitt & intercept \\
\hline multiple lineare Regression & multiple linear regression \\
\hline Verzerrung & bias \\
\hline adjustiert & adjusted \\
\hline Wechselwirkung & interaction \\
\hline Modellgüte & goodness-of-fit \\
\hline Regressionsdiagnostiken & regression diagnostics \\
\hline Bestimmtheitsmaß & coefficient of determination \\
\hline logistische Regression & logistic regression \\
\hline proportionales Hazards Modell & proportional hazards model \\
\hline
\end{tabular}

nein), kommt die logistische Regression (engl.: logistic regression), bei Überlebenszeiten [9] als Zielgröße das proportionale Hazards Modell von Cox in Frage. Auf diese Modelle werden wir in weiteren Artikeln eingehen $[2,10]$. Die englischen Bezeichnungen der hier diskutierten Begriffe zeigt Tab. 3.

\section{kurzgefasst}

Mit Hilfe der multiplen linearen Regression lassen sich Assoziationen zwischen einer stetigen Zielvariablen und mehreren erklärenden Variablen untersuchen. Der Regressionskoeffizient einer erklärenden Variable stellt ein nach den anderen Variablen adjustiertes Effektmaß dar.

Dieser Beitrag ist eine überarbeitete Fassung aus dem Supplement Statistik aus dem Jahr 2002.

\section{Literatur}

1 Altman DG, Bland JM. Comparing several groups using analysis of variance. BMJ 1996; 312: 1472-1473

2 Bender R, Ziegler A, Lange S. Logistische Regression. Dtsch Med Wochenschr 2007; 132: e33-e35

3 Draper NR, Smith H. Applied Regression Analysis (3rd Ed). Wiley, New York, 1998

4 Jr. Harrell FE, Lee KL, Mark DB. Multivariable prognostic models: Issues in developing models, evaluating assumptions and adequacy, and measuring and reducing errors. Stat Med 1996; 15: 361-387

5 Heise T, Kimmerle R, Heinemann L, Schubert H, Bender R, Pußkailer M, Berger $M$. Weight reduction in an out-patient obesity clinic: Which factors are associated with success? Int J Obes 1995; 19 (Suppl 2): 155

6 Lange S, Bender R. (Lineare) Regression/Korrelation. Dtsch Med Wochenschr 2007; 132: e9-e11

7 Lange S, Bender R. Was ist ein Signifikanztest? Dtsch Med Wochenschr 2007; 132: e19-e21

8 Ziegler A, Arminger G. Individualdaten-Regressionsanalyse. Vorlesungsskript (Kurs-Nr. 00 887). FernUniversität-Gesamthochschule Hagen, 2000

9 Ziegler A, Lange S, Bender R. Überlebenszeitanalyse: Eigenschaften und Kaplan-Meier Methode. Dtsch Med Wochenschr 2007; 132: e36-e38

10 Ziegler A, Lange S, Bender R. Das Cox-Modell. Dtsch Med Wochenschr 2007; 132: e39-e41 